\title{
Fuzzy Simulation of a Multi-electronic Acupuncture System and Clip-type Pulsimeter Equipped with a Magnetic Sensing Hall Device
}

\author{
You-Sik Hong ${ }^{1, \dagger}$, Jin-Kyu Rhee ${ }^{2, \uparrow}$, Han-Kyu Kim ${ }^{3}$, Il-Ho Son ${ }^{3}$, Woo-Sung Yoon ${ }^{3}$, Nam-Kyu Lee ${ }^{4}$, \\ Do-Young Park ${ }^{5}$, Keun-Ho Kim ${ }^{5}$, Yong-Jin Kim ${ }^{4}$, P. Khajidmaa ${ }^{4}$, and Sang-Suk Lee ${ }^{4,5 *}$ \\ ${ }^{1}$ Dept. of Computing Information and Engineering, Sangji University, Wonju 220-702, Korea \\ ${ }^{2}$ Western Seoul Center, Korea Basic Science Institute, Seoul 120-140, Korea \\ ${ }^{3}$ SMIC Co. Ltd., Gasan-dong, Geumcheon-Gu, Seoul 153-023, Korea \\ ${ }^{4}$ Dept. of Oriental Biomedical Engineering, Sangji University, Wonju 220-702, Korea \\ ${ }^{5}$ Dept. of Oriental-Western Biomedical Engineering, Sangji University, Wonju 220-702, Korea
}

(Received 15 July 2014, Received in final form 23 August 2014, Accepted 25 August 2014)

\begin{abstract}
A portable clip-type pulsimeter equipped with a magnetic sensing Hall device has been developed to raise the accuracy of oriental disease diagnosis and therapy by convergence of magnetism and oriental medicine. To improve accuracy and reliability of conventional pulsimeter due to subjective analysis of the pulse wave and measuring position dependency of the arterial pulse sensor, the fuzzy algorithm was applied to analyze the strong- and weak-pulse wave symptom. Optimal time for electronic acupuncture was calculated using fuzzy rules and interference were drawn from objective data in view of pre-treatment. Moreover, the electrical characteristics of the pain parts that respond to acupuncture point were applied in view of post-treatment to propose the scientific pulse wave algorithm and simulation experiment.
\end{abstract}

Keywords : permanent magnet, fuzzy rule, pulse wave detection system, systolic peak amplitude, BMI, notch time

\section{Introduction}

Pulse wave analysis is an important diagnostic index in Oriental medicine, and it is an important method of medical examination that judges the physical condition of a patient by information on the strength, size, and frequency as well as the character and state of the patient's pulse wave. However, such a method of pulse wave analysis has been regarded as a problem, in that the subjective decision of each Oriental doctor might mean that the physical condition of the same patient will be diagnosed differently depending on the doctor. Accordingly, it has been suggested that the instrument for pulse wave analysis in Oriental medicine should be objective in order to ameliorate the credibility of Oriental medicine and verify the reliability of Oriental medical treatment.

The existing pulsimeter was regarded as problematic, because it was not possible for anyone to be able to check

\footnotetext{
(C) The Korean Magnetics Society. All rights reserved.

These authors contributed equally.

*Corresponding author: Tel: +82-33-730-0415

Fax: +82-33-738-7610, e-mail: sslee@sangii.ac.kr
}

the pulse wave easily and conveniently at any time and in any place due to its heavy weight and bulky size $[1,2]$. Therefore, to ensure accurate pulse wave checking, the patient had to put up with the inconvenience of using a fixed-type pulsimeter set in an Oriental medicine clinic. Furthermore, the measurement result varies depending on the measuring location. This is because the existing pulsimeter cannot place the sensor exactly on the radial artery, and it has low reproducibility, as it is unusually sensitive to the measuring procedures used [3-5]. In addition, the traditional pulsimeter is regarded as having drawbacks, in that three-dimensional measurements cannot be carried out using the human hand, and it shows only the dimensional phenomenon of pulses [6-8]. In addition to this, the pulsimeter on sale currently is considered inconvenient to use, because it takes about 3 minutes to analyze the shape of the pulse wave even if the measuring sensor is located in the right place and the pressurizing conditions are all correct. That is, the clip-type pulsimeter is so inconvenient that it leads patients to raise objections to using it.

There is one more problem with it. When the blood pressure and pulse frequency are measured under fixed pressure without pressurization, the results are not accurate 
and reliable, because the pulse wave checking sensor cannot be placed exactly on the radial artery. This research aims to solve such problems by ameliorating the reproducibility of the clip-type pulsimeter and allowing the traditional pulse form to be measured intact by the pulsimeter. Thus, the researcher intends to develop a portable pulsimeter that makes it possible for anyone to measure the pulse wave easily at any time and any place [9-12].

In this research, the researcher has developed a prototype of a tiny clip-type portable pulsimeter equipped with a permanent magnet and a Hall device, as shown as Fig. 1(a), (b) and (c), breaking away from the existing bulky fixed-type pulsimeter that checks the pulse wave by way of pressurization. In this research, a clinical test was carried out in which the portable pulsimeter distinguished a strong pulse from a weak pulse, considering the systolic amplitude (rise waveform), reflected amplitude (reflected waveform), notch amplitude (notch waveform), rise time, reflective time, and notch point time. These were acquired by transferring one permanent magnet under steady pressure as well as by BMI (body mass index), blood pressure level, age, and gender [13-16].

In the research, the basic concept and theory of pulse wave analysis are examined for the scientification of Oriental medicine, and the principles and features of a pulsimeter designed based on the Hall sensor are examined $[17,18]$. The simulation results under fuzzy rules are examined to give the scientific basis for modernization of the pulsimeter system.

\section{Operating Principle of Clip-type Pulsimeter}

Though various types of pulsimeters have been developed, most of them have used the analogue method. Hence, it has been impossible to make a correct analysis of a patient's pulse wave. The pulse wave detection sensor should be placed exactly on the radial artery of the patient to correctly measure the pulse wave. However, it is very difficult for an Oriental doctor to place the sensor on the exact same part of the radial artery at every measurement. Therefore, the analogue-type pulsimeter cannot make a correct analysis. In addition to this, the existing pulsimeter cannot detect the patient's normal pulse waveform, since it pressurizes the whole wrist by the cuff when the patient's pulse wave is measured. When the patient's forearm is thick or thin, when the skin elasticity differs depending on the thickness of the blood vessels, when the patient is elderly, or when the patient is male, the pulse wave is especially difficult to measure exactly.

The basic concept behind discerning a strong pulse and (a)

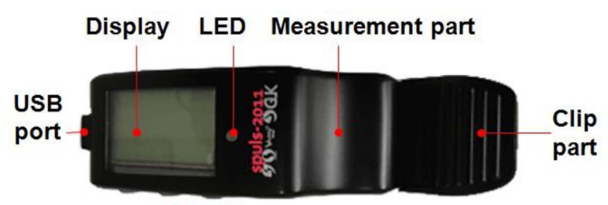

(b)

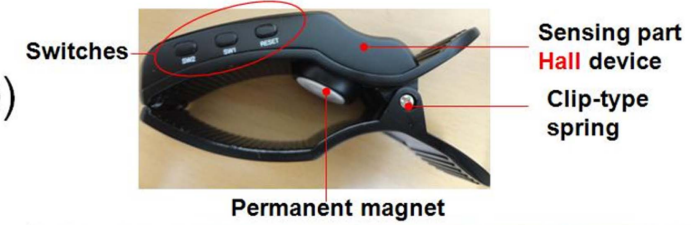

(c)

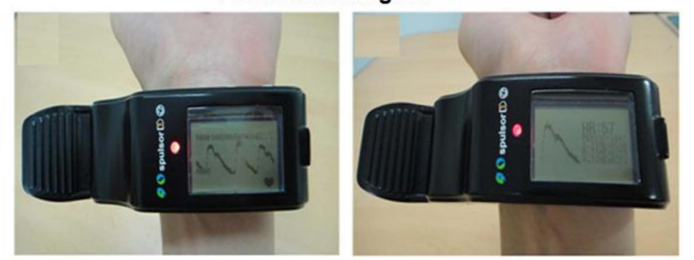

Fig. 1. (Color online) (a) Top view, (b) side view, and (c) appearance of pulse wave detection system for clip-type pulsimeter equipped with magnetic sensing Hall device.

a weak pulse is as follows. When the Oriental doctor checks the patient's pulse, some pulse waves feel strong and some pulse waves feel very weak. If the pulse of a healthy person feels strong, it is all right. However, if a patient with high blood pressure or diabetes has a strong pulse, it might suggest that the chronic disease has worsened. According to the beliefs of Oriental medicine, a weak pulse is generally found in those whose vitality is weak. In addition, according to the basic principle of pulse checking, the part (among the three parts of the radial artery Chon, Chwan, and Chuck) that shows the weakest pulse indicates that its relevant internal organ is especially weak.

Casting off the old way of thinking that a pulsimeter should be of the fixed type and in accordance with the trend of ubiquitous era, this thesis concentrates on developing a wearable wrist pulsimeter that anyone can use to measure the pulse wave simply and discern a strong pulse from a weak pulse while moving around. The Hall sensor principle used in this thesis follows below.

The Hall Effect is related with the electric charge that moves in a solid object or a leading wire where electrical current flows, as shown in Fig. 2(a). The magnetic field that exerts a transverse force on a conductor where electrical current flows pushes the moving electric charge to one side, and this phenomenon is called the Hall Effect. We can detect the direction of the force by applying Fleming's left-hand rule, stating that if a conductor through which an electric current flows is positioned perpendicular to the direction of a magnetic field, then that conductor will experience a mechanical force. Moreover, the 
(a)

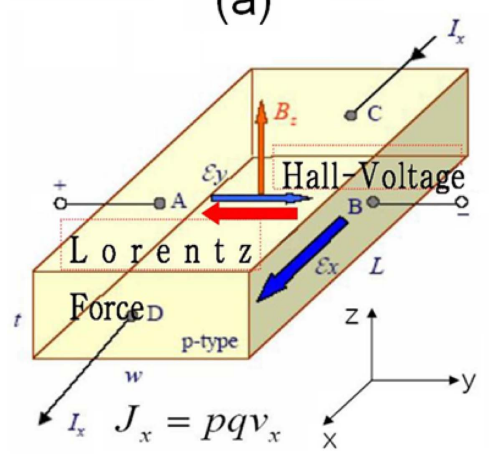

(b)

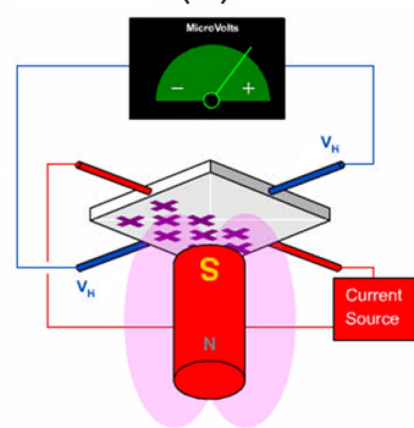

(c)

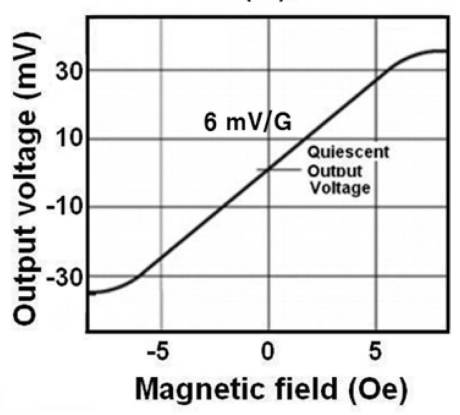

Fig. 2. (Color online) (a) Equilibrium state of electric force and magnetic force due to Hall voltage, (b) schematic of operating principle of one form of radial artery pulsimeter's pulse-sensing mechanism using Hall device and permanent magnet, and (c) output voltage versus magnetic field for commercial A3415-type Hall device with high sensitivity and linearity in magnetic field for clip-type pulsimeter equipped with magnetic sensing Hall device.

force level of the electric charge in objects can be measured by the Lorentz force rule. These two rules are the theoretical basis of the Hall Effect. If a particle of charge $q$ moves with velocity $\boldsymbol{v}$ in a magnetic field $\boldsymbol{B}$, it will experience a force that can be measured using Formula 1 below, as shown in Equation (1).

$$
\boldsymbol{F}_{B}=\mathrm{q} \boldsymbol{v} \times \boldsymbol{B}
$$

As seen in Fig. 2(a), the Hall voltage that corresponds with the Lorentz force makes the electric charge in the magnetic field flow straight without bending, and the force generated by the Hall voltage in the electrical field is balanced by the Lorentz force by the magnetic field, owing to this Hall Effect. When a permanent magnet is attached to the skin's surface near the radial artery in order to measure the radial pulse, the location of the magnet permanent changes following the perpendicular displacement of the radial artery, and the intensity of the magnetic field felt through the sensor at some distance changes at this time.

Fig. 2(b) shows the schematic of the operating principle of one form of the radial artery pulsimeter's pulse-sensing mechanism by using one Hall device and a permanent magnet. The sensor signal concerning the magnetic field in such changes is converted to a voltage signal, and at the time of this signal change, the mixed noise signal is converted as well. Fig. 2(c) shows the output voltage versus the magnetic field for a commercial A3415-type Hall device with a high sensitivity and linearity in the magnetic field for the clip-type pulsimeter equipped with a magnetic sensing Hall device. Therefore, the converted signal should be filtered through an analogue filter, and the filtered signal is to be sent to a processor with a builtin $\mathrm{A} / \mathrm{D}$ converter after being amplified. Then, the A/D converter accumulates the digitized signal after digitalizing the analogue signal. Regarding pulsation, the maximum vertical displacement per waveform unit is about 1.13 $\mathrm{mm}$. Therefore, the maximum displacement of a magnet attached to the skin should be within about $1.13 \mathrm{~mm}$. An $\mathrm{Nd}$ magnet with a thickness of $1 \mathrm{~mm}$ and a diameter of 3 $\mathrm{mm}$ was selected in this research, as it is affordable, easily attached to the skin's surface, and easily attached to the part to be measured $[19,20]$.

\section{Analysis of Pulse Waveform}

In Oriental medicine, doctors must accurately discern substantial symptoms and mild symptoms in order to cure the disease of a patient. When a patient suffers from a cold in the early stages, the doctor uses a cleansing medicine, judging it as a substantial symptom. In the beginning, the patient becomes feverish, feels his whole body aching, and has a cough or a runny nose. From these symptoms, the Oriental doctor would suppose that the patient's body is full of ill energy (energy of disease), and he would use a cleansing medicine to cure it. It is not desirable to use a cleansing medicine for a long time.

Occasionally, some patients suffer from long-term colds without taking a turn for the better, and they are apt to feel tired and have hacking coughs with no salient symptoms. In this case, in accordance with the principles of Oriental medicine, a restorative medicine should be prescribed instead of a cleansing medicine.

The pulse waveform should be changed especially when one feels a sensation of fear or worry. In the case of female patients, the pulse waveform may vary significantly during the periods of menstruation. Therefore, doctors should consider the physical condition and mental state of 


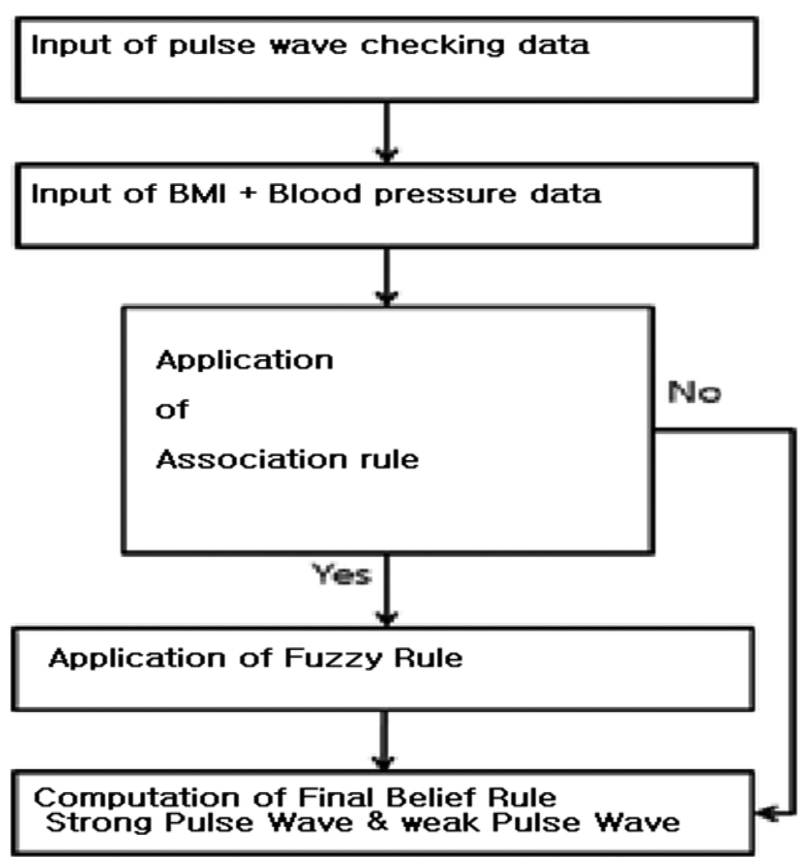

Fig. 3. Flowchart of intelligence pulse wave system using fuzzy rules.

each patient to make a correct and reliable analysis of pulse waveform even when the best pulsimeter is used. In an attempt to ameliorate the situation explained above, the researcher aims to enhance the reliability of the portable clip-type pulsimeter by using the Hall sensor to realize an intelligence algorithm. Since doctors should consider the patient's physical condition, age, and gender, the flowchart of the intelligence pulse wave system using fuzzy rules based on this study suggests an algorithm where the belief value regarding the disease affirmation is calculated, as shown in Fig. 3.

\section{Simulation Using Fuzzy Rules}

First, in order to get the conditional probability $\mathrm{P}(\mathrm{Hi} \mid \mathrm{E})$, the prior probability $\mathrm{P}(\mathrm{Hj})$ and the conditional probability $\mathrm{P}(\mathrm{E} \mid \mathrm{Hj})$ should be known. For example, $\mathrm{P}(\mathrm{E} \mid \mathrm{Hj})$, the probability that the symptom of each disease may upspring, should be given when it is inferred that $\mathrm{E}$ is the symptom presenting in the patient and $\mathrm{Hi}$ is the disease. However, in reality, data for such things are quite often insufficient. Therefore, the belief value regarding disease affirmation is calculated using the fuzzy rule, as seen below.

Preconditioning RULE: (True Pulse)

IF $\mathrm{S}$ amp (amplitude) is maximum

OR $\mathrm{S}$ time of patient's pulse is maximum

AND $\mathrm{N}$ time of patient's pulse is maximum
AND R time of patient's pulse is maximum

OR R amp of patient's pulse is maximum

OR N amp of patient's pulse is maximum

Patient's pulse is not "Hongmaeck- a type of pulse"

Patient's pulse is not "Ginmaeck- a type of pulse"

Patient's pulse is not "Noemaeck- a type of pulse"

THEN it is a true pulse.

Figure 3 explains how the fuzzy rule and the belief value obtained through statistic-based probabilistic data are used in the final process for diagnosing a disease if it is difficult to make a correct inference due to some physical conditions when more than two data values from pulse wave checking and tongue diagnosis need to be inferred to measure the patient's disease more accurately.

Rule: IF A is t1, THEN C is B2

(Fu)

Fact: A is $\mathrm{t}^{\prime}$ ( $\mathrm{Fr}$ )

Conclusion: $\mathrm{C}$ is $\mathrm{t}^{2}$ '

(FC)

A: Condition of clinical patient

C: Result of inference

Fu: Fuzzy number showing the uncertainty of the rule

Fr: Fuzzy number showing the uncertainty of the fact

FC: Fuzzy number showing the uncertainty of the conclusion

V1, V2, V1', V2': values

$\mathrm{Fu}$ shown above is represented by Confidence factor (CF), and Fr is represented by probability scale.

Post-processing RULE (strong wave)

IF $S$ amp $=$ Med And

$\mathrm{BMI}=$ High And

H_Blood $=$ High And

$\mathrm{N}$ time $=$ Med

Then

Strong wave $=\mathrm{CNF} 70$

Here, CNF signifies that the certainty of a strong (excess syndrome) wave rule is $70 \%$. The probability that a patient is diagnosed as having a strong wave is always $100 \%$ if we display the certainty factor following the traditional method with no fuzzy rule included. The reliability of a strong wave is set at $70 \%$ in this thesis, considering the M BP (maximum blood pressure), S amp (rise waveform), $\mathrm{N}$ time (notch point time), and BMI (weight $(\mathrm{kg}) /$ height $\left(\mathrm{m}^{2}\right)$ ). If a user gives a certainty of $80 \%$ to the fact that $\mathrm{S}$ amp $=$ Low, the degree of certainty regarding the conclusion becomes $0.56(0.8 \times 0.7=0.56)$. It is very difficult to extrapolate a disease by checking the pulse waveform, because the velocity and intensity of the 
Table 1. Input data to discern strong (excess) syndrome waves from weak (deficiency) syndrome waves.

\begin{tabular}{cc}
\hline \hline \multicolumn{2}{l}{ Input data to discern strong syndrome waves from weak syndrome } \\
waves
\end{tabular}

pulse wave may differ depending on the gender, age, and physical condition of the patient. Therefore, this researcher used the belief value matching function, which is used in recalculating the belief value when there are more than two estimating values concerning the same disease.

As seen in Table 1 above, any one of the 10 variables can change the data of the pulse waveform, even if the pulse waveform is checked from the same patient. In order to eliminate such a problem, the researcher tried to analyze the pulse waveform more accurately, reflecting 10 variables in this research.

Fig. 4 explains the 32 fuzzy rules and fuzzy output membership used in this thesis. According to the explanation given in Fig. 5, the probability that a patient has a strong (syndrome wave) pulse is $93 \%$ if his BMI, high blood pressure, $\mathrm{S}$ amp, and $\mathrm{N}$ time values are 29, 174, 2250 , and 336, respectively. In a simulation performed in this research, it was judged that the patient had a weak pulse if his $\mathrm{S}$ amp and $\mathrm{N}$ time values were 1900 and 310, respectively, even though he had the same physical condition as above.

Fig. 5 shows the multipad with a built-in electronic acupuncture device. Changes in a person's age, gender, humidity, temperature, weight, and fat accumulation are

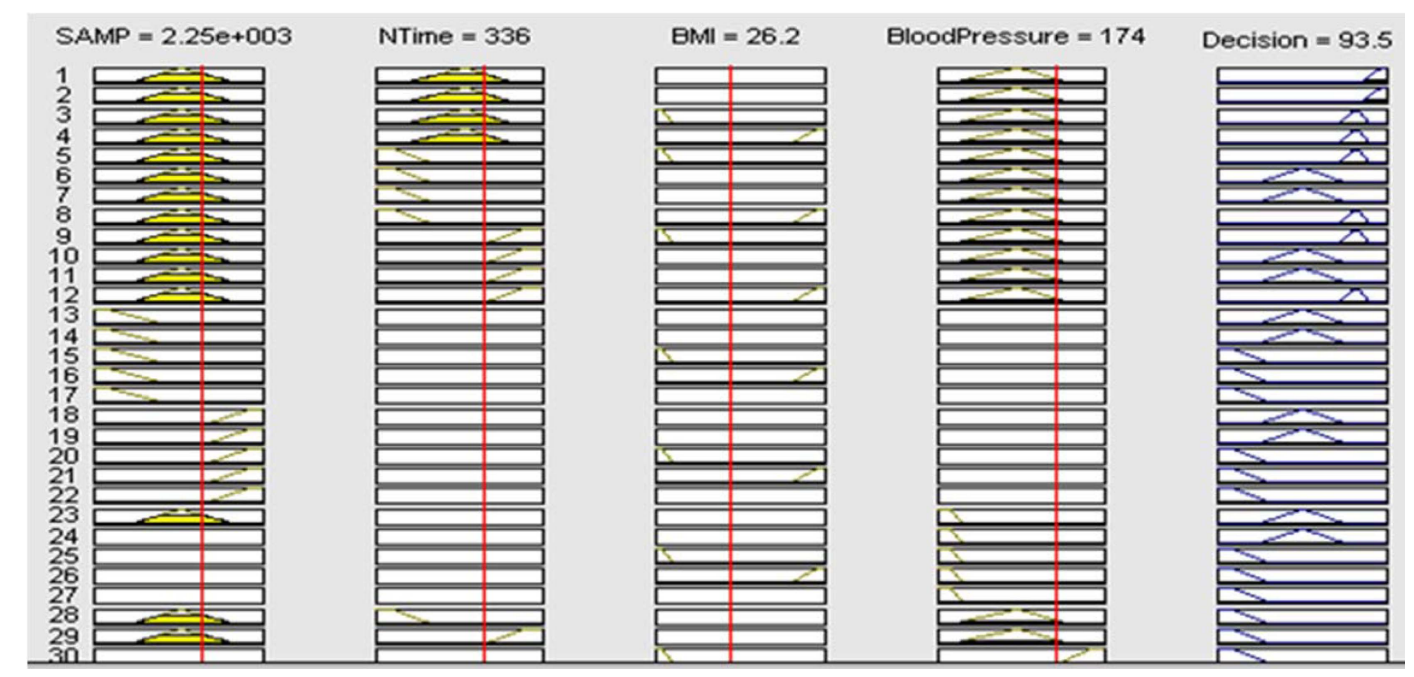

Fig. 4. (Color online) Fuzzy membership input functions and FIS(fuzzy inference system) rules.
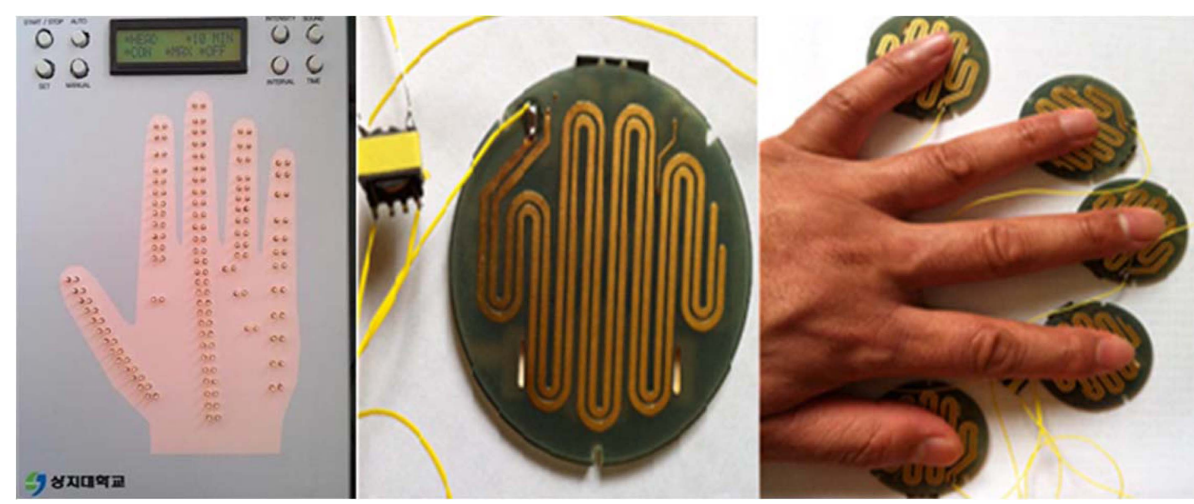

Fig. 5. (Color online) Multipad with built-in electronic acupuncture device. 
based on the changes. In this paper, in order to solve this kind of problem, a compositional inference rule was used while using the fuzzy rule. The fuzzy compositional rule of inference was applied to come up with a calibrating constant in order to derive an accurate result (considering the patient's physical condition) in analyzing the blood pressure pulse.

\section{Conclusion}

In Oriental medicine, a variety of diseases are diagnosed and treated by analyzing the pulse wave of patients. Until now, the method of pulse wave analysis using the pulsimeter has been considered subjective. Therefore, the existing method of pulse wave analysis has been criticized due to its low reliability. Furthermore, the measurement result varies depending on the measuring location, as the existing pulsimeter cannot place the sensor exactly on the radial artery, and the existing pulsimeter has low reproducibility, as it is unusually sensitive to the measuring procedures used. In this research, it was suggested that the strong pulse and the weak pulse could be discerned by using a portable pulsimeter designed based on a magnetic sensing Hall device to eliminate the problems mentioned above. The optimal time for electronic acupuncture was calculated using fuzzy rules. In order to calculate the exact acupuncture time, physical condition, illness, and age were considered. However, this classification scheme depending on the patient's illness conditions varies greatly. Parametric consideration of difference among patients' physical conditions could objectify analysis of diagnostic data and management of treatment of oriental doctor by this pulsimeter equipped with magnetic sensing Hall device.

\section{Acknowledgement}

This research was supported by the MSIP (Ministry of Science, ICT and Future Planning), Korea, under the IT/ SW Creative research program supervised by the NIPA
(National IT Industry Promotion Agency) (NIPA-2013H0502-13-1112).

\section{References}

[1] G. G. Yu, Pulse wave detection, Modern Acupuncture Hospital (2006) pp. 86-105.

[2] I. B. Wilkinson, S. A. Fuchs, I. M. Jansen, J. C. Spratt, G. D. Murray, J. R. Cockcroft, and D. J. Webb, Hypertension 16, 2079 (1998).

[3] J. Y. Kim and J. K. Inst, Ori. Med. 14, 81 (2008).

[4] S. S. Lim and K. J. Lee, J. Biomed. Eng. Res. 21, 411 (2000).

[5] J. W. Hong, Ocular inspection and pulse wave detection, Wonil Press (2000) pp. 256-272.

[6] J. W. Kim, S. G. Jung, and D. Y. Kim, J. Kor. Acad. Indu. Coop. Soc. 13, 5005 (2012).

[7] Y. S. Hong, J. Inst. Inter. Broad. Comm. 13, 245 (2013).

[8] M. C. P. Carrasquero, L. M. H. Bemard, H. Kobori, Y. Ozawa, K. S. H. Smith, L. L. Hamm, and L. G. Navar, Hypertension 44, 223 (2004).

[9] Clinic hospital website, http://www.kykhani.com

[10] B. Flaws, The Secret of Chinese Pulse Diagnosis, Boulder, Blue Poppy Press (1995) pp. 13-18.

[11] H. H. Dao, M. L. M. L. Frelut, F. Oberlin, G. Peres, P. Bourgeois, and J. Navarro, Inter. J. Obesity 28, 290 (2004).

[12] Y. S. Hong, ICHIT, LNCS 6935, Springer, 213 (2011).

[13] S. Walsh and E. King, Pulse diagnosis: A clinical guide, Piladelphia, PA: Elsevier Limited (2008) pp. 45-55.

[14] M. L. Garg, S. I. Ahson, and D. V. Gupta, Infor. Pro. Lett. 39, 165 (1992).

[15] K. S. Leung and W. Lam, IEEE Comp. International Symposium, 43 (1988).

[16] G. C. Looney and A. A. Alfize, IEEE Trans. SMC 17, 1077 (1987).

[17] D. H. Nam, W. B. Lee, Y. S. Hong, and S. S. Lee, Sensors 13, 4714 (2013).

[18] J. K. Rhee and S. S. Lee, J. Magn. 18, 183 (2013).

[19] S. S. Lee, I. H. Son, J. G. Choi, D. H. Nam, Y. S. Hong, and W. B. Lee, J. Korean Phys. Soc. 58, 349 (2011).

[20] S. S. Lee, J. G. Choi, I. H. Son, K. H. Kim, D. H. Nam, Y. S. Hong, W. B. Lee, D. G. Hwang, and J. R. Rhee, J. Magn. 16, 449 (2011). 